
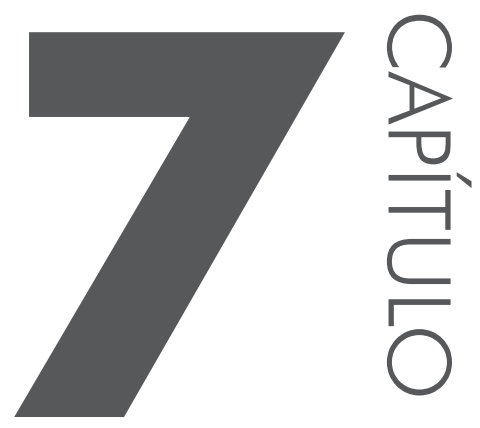

\title{
UM MODELO DE DADOS \\ VOLTADO AO SERVIÇO \\ DE INTELIGÊNCIA POLICIAL
}

Herbson de Carvalho

Alexandre Leopoldo Gonçalves

\section{INTRODUC̣ÃO}

Segundo o Dicionário Michaelis, o vocábulo inteligência, do latim intelligentia, significa a faculdade de entender, pensar, raciocinar e interpretar; entendimento, intelecto; o que se pode entender também por habilidade para escolher os métodos e obter um bom resultado. Neste trabalho, o foco reside na atenção à inteligência policial, que é atividade realizada pelo Estado por meio de suas instituições policiais. As instituições tradicionalmente responsáveis por realizarem as atividades de inteligência são conhecidas como Serviços de Inteligência e definidas por Cepik (2003, p. 13) como: “[...] agências governamentais responsáveis pela coleta, pela análise e pela disseminação de informações consideradas relevantes para o processo de tomada de decisões e implementação de políticas públicas nas 
áreas de política externa, defesa nacional e provimento de ordem pública”.

De acordo com Martins (2011), inteligência, no contexto policial, é entendida como uma atividade proativa, caracterizada pela busca de informações, com a finalidade de possibilitar ao gestor público um supedâneo mínimo para seu poder decisório. Com base em informações contextualizadas, o destinatário final da atividade de inteligência poderá propor e comandar ações concretas em termos de segurança pública.

No Brasil, as instituições policiais, sejam elas civis, militares ou federais, possuem em suas estruturas serviços de inteligência responsáveis pela coordenação e realização das atividades afins. Segundo Pacheco (2005), o serviço de inteligência policial pode ser subdividido em três áreas: análise, contra-inteligência e operações.

As instituições que atuam em segurança pública, em especial as polícias militares, possuem, entre seus departamentos, Agências de Inteligência (AI) que integram do Sistema Brasileiro de Inteligência (Sisbin). Os órgãos de inteligência possuem como atividade o exercício de ações especializadas para identificação, acompanhamento e avaliação de ameaças reais ou potenciais na esfera da segurança pública. Atuam ainda na obtenção, produção e salvaguarda de dados, informações e conhecimentos que subsidiem ações da Polícia Militar para neutralizar, coibir e reprimir atos criminosos de qualquer natureza.

Um dos principais desafios para as pessoas e para as organizações é saber detectar e gerenciar a informação de maneira eficaz, conforme citado por Lira et al. (2008), pois a busca e coleta de dados para a produção de conhecimento gera uma grande quantidade de dados e informações. O enorme volume de dados acaba, muitas vezes, por dificultar a produção de um conhecimento, ao passo que exige que cada analista tenha que rever e confrontar diversos dados de diversas fontes diferentes.

Para tentar organizar e armazenar esses dados, faz-se necessário o uso de sistemas que possam tornar a grande quantidade de dados existentes em informação. Adiciona-se ainda a necessidade de aprimorar determinado contexto por meio da transformação de informações em conhecimento. Sistemas são importantes para auxiliarem os gestores na tomada de decisão. No caso da inteligência policial, a utilização de sistemas informatizados são uma ferramenta para essencial para o analista.

O ponto de partida para este trabalho considera que cada AI adota sua maneira de armazenar dados sobre potenciais alvos de investigação. Esses alvos podem ser indivíduos, veículos ou locais. Em muitos casos, são confeccionados Relatórios de Inteligência em formato de texto e repassados por meio de mensagens eletrônicas, sendo arquivados em computadores. O principal desencontro de informações é que cada AI arquiva seus documentos de uma forma. Pode-se 
dizer que cada agência busca maneiras individuais de organizar melhor seus dados e informações. Esse problema é recorrente em diversos órgãos de inteligência. Gabrielli (2007), em seu trabalho sobre os núcleos de inteligência da Polícia Rodoviária Federal de Santa Catarina, destaca que, apesar da grande quantidade de atribuições dos núcleos, eles não possuem sistemas que realmente possam auxiliar em suas atividades.

Adiciona-se a isso o fato de que parte da informação sobre um indivíduo está, no máximo, apenas na memória do agente que participou de determinada operação. Muitas vezes, um agente coletou dados e informações acerca da mesma pessoa em diferentes momentos e isto poderia auxiliar na identificação de ações criminosas, mas é desconhecido por outros agentes que possuem o mesmo alvo. Em muitos casos, não há sequer um banco de dados que reúna informações sobre esses indivíduos, como locais que frequenta, pessoas com quem se relaciona, veículos que utiliza e outros dados. O mais comum é a existência de banco de dados dispersos, que armazenam somente ocorrências policiais incompletas.

Neste sentido, existe uma lacuna nos órgãos de inteligência, ou seja, a inexistência de um banco de dados sobre informações adquiridas pelos agentes. Um banco de dados com tais características poderia armazenar esses dados e informações, compartilhando com as demais agências, que teriam acesso ao conhecimento já produzido por um conjunto de agentes de inteligência. Isso resultaria, claramente, em uma redução de trabalho e em mais qualidade nos relatórios produzidos, auxiliando no processo de tomada de decisões com maior precisão.

Neste sentido, este trabalho tem por objetivo propor um modelo de dados capaz de promover subsídios à tomada de decisão no contexto da inteligência policial, bem como demonstrar o potencial por meio de exemplos de sua utilização.

\section{MODELO PROPOSTO}

O presente trabalho propõe um modelo de dados, sendo este uma representação de determinada realidade, domínio ou contexto. Pretende-se, a partir da proposta, apresentar os principais elementos para suportar a captura e o armazenamento de dados no contexto da inteligência policial.

Inicia-se pela modelagem, ou seja, pelo processo de representar o mundo observado real ou imaginário (COUGO, 1997) considerando um conjunto de requisitos. Em seguida, tais requisitos devem ser apresentados em uma linguagem que permita analisar mais facilmente os componentes do domínio, bem como os relacionamentos entre os mesmos. Para tal, a modelagem conceitual caracteriza-se como um ferramental capaz de transcrever as necessidades em um diagrama com o intuito de deixar claro o que se deseja com o modelo de dados. 
A modelagem conceitual concentra-se em um nível de abstração e deve ser usada para envolver o usuário final, pois o objetivo é discutir os aspectos do banco de dados e não da tecnologia. Os exemplos de modelagem de dados vistos ao nível do modelo conceitual são mais fáceis de compreender, já que não há limitações ou aplicação de tecnologia específica.

Segundo Heuser (2009), o modelo conceitual captura as necessidades da organização em termos de armazenamento de dados independentemente de implementação. A modelagem conceitual é o primeiro estágio para um projeto de banco de dados que visa suportar sistemas capazes de gerir os dados e, a partir destes, fornecer informações que possam ser utilizadas na tomada de decisão. Após a modelagem conceitual, tornam-se necessárias as modelagens lógica e física, estando estas mais voltadas ao próprio banco de dados.

Existem muito modelos ou tipos de bancos de dados. Entre os mais utilizados atualmente encontra-se o modelo relacional. Um banco de dados relacional é um mecanismo de armazenamento que permite a persistência de dados e, opcionalmente, a implementação de funcionalidades. O que caracteriza o modelo relacional é a maneira como os dados são armazenados e organizados no banco de dados. No contexto deste trabalho, o foco está voltado aos Sistemas de Gerenciamento de Banco de Dados Relacional (SGBDR - do inglês Relational Database Management System [RDBMS]), que se apresentam ou são percebidos pelo usuário na forma de tabelas (GRAD, 2012; CAMPBELL-KELLY, 2012).

\subsection{Modelagem conceitual}

Pensando na criação de um banco de dados voltado ao uso na inteligência policial, a primeira entidade a ser criada é a Informacao. Esta entidade contém um atributo identificador (atributo identificado por uma bolinha azul), além dos campos de texto Descricao, no qual será descrito o dado/informação que se deseja armazenar. O campo DataFato é responsável por armazenar a data em que ocorreu o descrito na informação. Existem ainda os campos TipoInformacao e DataCadastro, que armazenaram, respectivamente, o tipo de informação e a data do cadastro.

Outras quatro entidades são necessárias para completar a entidade Informacao, sendo elas: Veiculo, Individuo, Geo e Ocorrencia. A entidade Veiculo é responsável por armazenar os dados referentes a veículos, como Placa, MarcaModelo, Ano, Cor e Chassi. O atributo identificador é determinado por meio do atributo IdVeiculo.

A entidade Individuo armazena dados sobre pessoas, tendo como atributo identificador IdIndividuo. Já a entidade Geo é responsável por armazenar dados sobre locais como endereços e coordenadas geográficas, tendo como atributo identificador IdGeo. A entidade Ocorrencia armazenará todos os tipos de ocor- 
rências policiais existentes. A Figura 7.1 apresenta o modelo conceitual proposto neste trabalho.

A entidade Informacao poderá ter mais de um indivíduo e mais de um veículo relacionado, bem como, pode estar associado a mais de um local e mais de um tipo de ocorrência. Também pode existir um relacionamento com outra tupla cadastrada anteriormente. Isto ocorre através do conceito de auto-relacionamento, representado no modelo por um losango anexo a própria entidade Informacao.

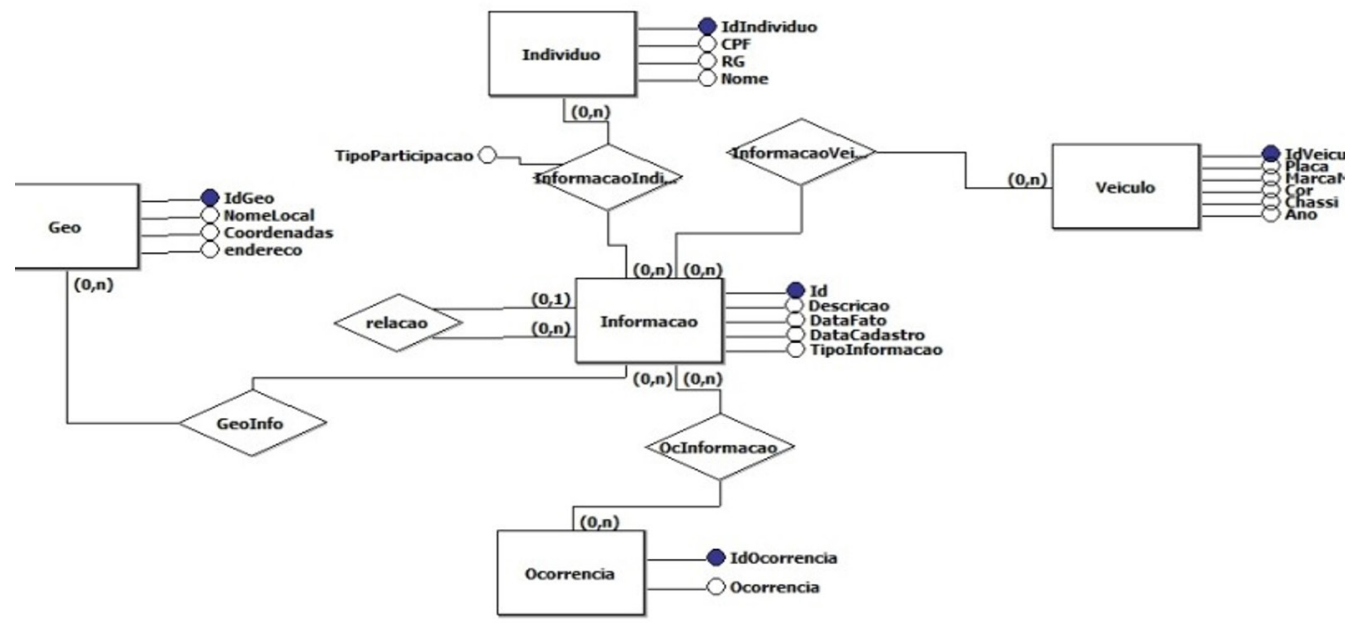

Figura 7.1 Modelo conceitual proposto.

\section{UTILIZAC̣ÃO DO MODELO}

O cenário proposto para a avaliação do modelo é baseado nos órgãos de inteligência da Brigada Militar (BM) do Estado do Rio Grande do Sul. Para dimensionar o tamanho real que o modelo proposto de banco de dados poderia trabalhar, utiliza-se o número de ocorrência do ano de 2014, com base nas informações fornecidas pela Secretária de Segurança Pública do Estado do Rio Grande do Sul (SSP/RS). Conforme dados estatísticos da SSP em atendimento à Lei $n^{\circ} 11.343 / 99$ e à Lei $n^{\circ} 12.954 / 08$, em 2014, a BM/RS realizou 759.715 atendimentos de crimes consumados e 20.458 tentativas de crimes (RS, 2014).

Neste estudo, foram incluídos alguns dados para possibilitar a simulação da utilização do banco de dados. Sendo assim, foram cadastrados 1.425 relatórios de inteligência, 21 indivíduos, sete veículos e 519 locais de ocorrências.

Esses dados foram utilizados como forma de permitir a utilização de uma aplicação que possibilitasse, a partir de consultas, demonstrar a viabilidade do modelo proposto. A quantidade de dados, conforme mencionado, foram cadas- 
trados com base no número médio de ocorrências de um período de três meses em apenas uma cidade de pequeno porte.

\subsection{Exemplos de consultas e discussões}

A partir do modelo proposto é possível realizar diversas consultas. Com a finalidade de facilitar a demonstração dos resultados que podem ser obtidos, foi desenvolvida uma aplicação em Linguagem PHP ${ }^{\circledR}$ (PHP: Hypertext Preprocessor). A seguir, serão demonstrados e discutidos alguns resultados obtidos a partir do modelo proposto e povoado conforme descrito anteriormente.

Entre as possíveis resultados, cita-se a possibilidade de relacionar todas as informações de determinado indivíduo, apresentando também quais outras pessoas foram citadas conjuntamente. Demonstra ainda os veículos e os locais identificados com um indivíduo em questão (Figura 7.2).

\section{Individuo}

\begin{tabular}{|l|l|}
\hline Nome & Mario J Junior \\
\hline Alcunha & Mario \\
\hline RG: & I \\
\hline CPF: & \\
\hline
\end{tabular}

Relatório onde é citado:

\begin{tabular}{|c|c|c|c|}
\hline Relatório & Fato & Participaçao & Data \\
\hline Registro Policial n० $1.366 / 2015$ & Fate em tese Atioice. & Só comunicante & $26 / 04 / 201514: 30: 00$ \\
\hline Registro Policial no $1.364 / 2015$ & Exercicie arbitrárie da própna razães & Acusado & $26 / 04 / 2015 \quad 12: 00: 00$ \\
\hline Registro Policial n $1.406 / 2015$ & Iniúria, & Nitima & $14 / 10 / 201418: 30: 00$ \\
\hline Registro Policial no $1.402 / 2015$ & Desobedièncias & Acusado & $14 / 10 / 201418: 30: 00$ \\
\hline Registro Pclicial no $1.373 / 2015$ & Qutros Crimes, & Acusado & $11 / 08 / 200915: 00: 00$ \\
\hline Registro Pclicial n 1.394/2015 & Lesāo Corporal Culposa Art.303. & Autor & $18 / 11 / 200814: 00: 00$ \\
\hline Registro Pclicial n $1.356 / 2015$ & Retencão de veiculo, & Acusado & $03 / 05 / 200716: 00: 00$ \\
\hline Registro Pclicial nº 1.378/2015 & Perda de Documento, & Só comunicanse & $01 / 12 / 1999$ 0C:01:00 \\
\hline Registro Pclicial n $1.365 / 2015$ & Dinicir s/habilitacão Art. 309. & Só comunican:e & $09 / 09 / 199912: 45: 00$ \\
\hline Registro Pclicial n० 1.411/2015 & Diricir s/habilitacão Art.309, & Adolescente infrator & $07 / 04 / 199822: 00: 00$ \\
\hline
\end{tabular}

Pessoas que foram citadas junto com este individuo:

\begin{tabular}{|c|c|c|}
\hline Pessoa & RG & Quantidade \\
\hline Roberto $\mathrm{N}=\mathrm{\text {(Beto) }}$ & RG: $=$ & $2 x$ \\
\hline Fabiano C $=$ (Camelo) & 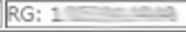 & $1 x$ \\
\hline
\end{tabular}

Locais onde foi citado:

\begin{tabular}{|cc|c|}
\hline Local Qndereco & Quantidade \\
\hline
\end{tabular}

Veiculos com os quais foi citado:

\begin{tabular}{|c||l|l|}
\hline Placa & Marca Modelo - Cor & Quantidade \\
\hline \hline KEE-75s & Ford Ranger 13d - Azul & \\
\hline \hline
\end{tabular}

Figura 7.2 Relatório de informações do indivíduo. 
Como todos os registros possuem informações sobre a localização em que ocorreu o fato, é possível, com base nesta mesma pesquisa, exibir um mapa que mostrará os locais por onde este indivíduo passou (Figura 7.3). Para produzir o mapa, é necessário recuperar os registros nos quais foi citado o indivíduo em questão e resgatar os dados do endereço que foram cadastrados junto àquele registro. Os dados referentes à localização estão disponíveis na tabela Geo, na qual é efetuada a consulta para recuperar a informação do endereço e, utilizando ferramentas de georeferenciamento, apresentar um mapa.

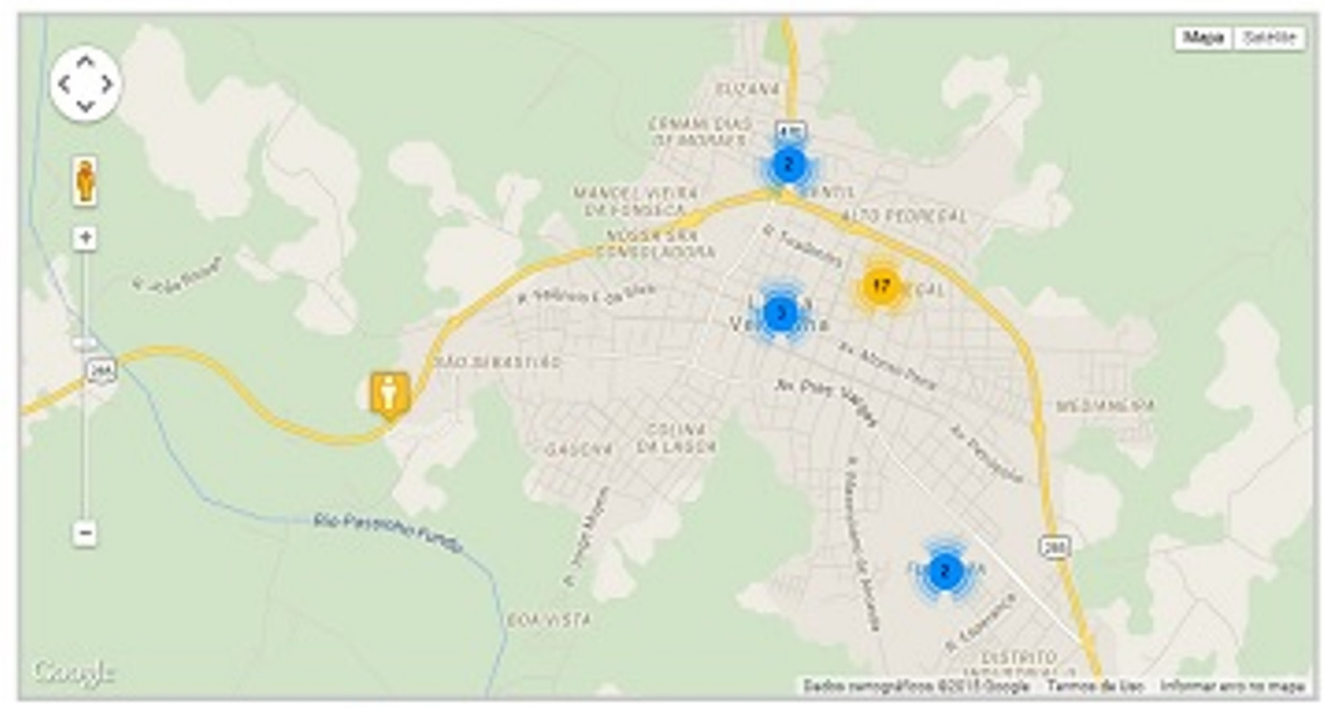

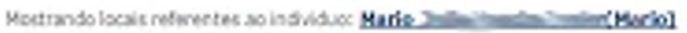

Figura 7.3 Mapa de ocorrências de indivíduo.

Estas duas representações (relatório de informações agregadas e mapa de circulação de determinado indivíduo) obtidas a partir do banco de dados podem ser vistas como relevantes para o uso na inteligência policial, pois, com elas, torna-se possível visualizar, de forma fácil, todos os registros de informações que foram efetuados relacionando determinado alvo.

\section{CONSIDERAC̣ÕES FINAIS}

O objetivo geral deste trabalho foi o desenvolvimento de um modelo de banco de dados para a aplicação na gestão da informação com foco em ações de inteligência policial. Neste sentido, foi um proposto o modelo de dados detalhando os principais elementos capazes de criar uma especificação ao desenvolvimento de sistemas de informação voltados à inteligência policial. Especificou-se o modelo 
conceitual, sendo este o primeiro passo para a criação dos esquemas de relações que no banco de dados são implementadas como tabelas.

Após a concepção do banco de dados, foi elaborado um cenário envolvendo um conjunto de relatórios de inteligência, indivíduos, veículos e locais de ocorrência. O cenário teve como objetivo permitir a execução de consultas e a obtenção de informações consolidadas. Para tal, foi desenvolvida uma aplicação ao nível de protótipo capaz de apresentar, a partir de determinado indivíduo de interesse, informações direta ou indiretamente relacionadas. Essas informações visam auxiliar no entendimento do contexto de inserção de determinado indivíduo alvo.

Os resultados obtidos, ainda que iniciais, demonstram que o modelo de banco de dados proposto pode ajudar muito na confecção de Relatórios de Inteligências por parte dos agentes. De certo modo, isso promove mais credibilidade aos relatórios e mais segurança ao tomador de decisão. A agregação da informação, bem como a confiabilidade da informação utilizada na tomada de decisão, pode produzir melhores resultados e, por consequência, melhorar a efetividade das ações de inteligência policial.

\section{REFERÊNCIAS}

CAMPBELL-KELLY, M. The RDBMS Industry: A Northern California Perspective. IEEE Annals of the History of Computing, v.34, n.4. 2012.

CEPIK, M. A. C. Espionagem e Democracia: agilidade e transparência como dilemas na institucionalização de serviços de inteligência. Rio de Janeiro: FGV, 2003.

COUGO, P. Modelagem conceitual e projeto de bancos de dados. Rio de Janeiro: Elsevier, 1997.

GABRIELLI, B. V. Desenvolvimento de um sistema de apoio a inteligência policial. Departamento de Informática e Estatística - Universidade Federal de Santa Catarina (UFSC). 2007. Disponível em: <https://projetos.inf.ufsc.br/arquivos_projetos/ projeto_681/artigoVentorim.pdf>. Acesso em: 27 abr. 2015.

GRAD, B. Relational Database Management Systems: the formative years. IEEE Annals of the History of Computing, v.34, n.4, 2012.

HEUSER, C. A. Projeto de banco de dados. 6.ed. Porto Alegre: Bookman, 2009. LIRA, W. S.; CANDIDO, G. A.; ARAÚJO, G. M.; BARROS, M. A. A busca e o uso da informação nas organizações. Perspect. ciênc. inf. [online]. v.13, n.1, 2008. ISSN 14139936. Disponível em: <http://dx.doi.org/10.1590/S1413-99362008000100011>. Acesso em: 26 abr. 2015.

MARTINS, A. F. Inteligência policial e investigação. 2011. Disponível em: <https:// blogdodelegado.wordpress.com/2011/04/13/inteligencia-policial-e-investigacao/>. Acesso em: 10 mar. 2015. 
PACHECO, D. F. Atividades de inteligência e processo penal. IV Jornada Jurídica da Jurídica da Justiça Militar da União. Auditoria da $4^{\text {a }}$ CJM. Juiz de Fora, 30 set. 2005. Disponível em: <http://www.advogado.adv.br/direitomilitar/ano2005/ denilsonfeitozapacheco/atividadedeinteligencia.htm>. Acesso em: 15 dez. 2014.

RIO GRANDE DO SUL - RS. SECRETARIA DA SEGURANÇA PÚBLICA. DEPARTAMENTO DE GESTÃO DA ESTRATÉGIA OPERACIONAL. Sistema Estatístico da SSP/RS. Dados estatísticos da Secretaria da Segurança Pública em atendimento à Lei no $11.343 / 99$ e à Lei no $12.954 / 08$, contendo os dados do primeiro semestre de 2014. 2014. Disponível em: <http://www.ssp.rs.gov.br/ upload/201409111110442014s01_lei_postal_e_lei_stela_farias_1_semestre_de_2014. pdf>. Acesso em: 26 abr. 2015. 
